\title{
Trivium
}

Revue franco-allemande de sciences humaines et sociales - Deutsch-französische Zeitschrift für Geistesund Sozialwissenschaften

$3 \mid 2009$

Droits subjectifs et droits de l'homme

\section{L'interprétation des droits de l'homme : enjeux politiques et théoriques au prisme du débat français}

\author{
Catherine Colliot-Thélène
}

\section{OpenEdition}

Journals

Édition électronique

URL : http://journals.openedition.org/trivium/3290

DOI : $10.4000 /$ trivium.3290

ISSN : 1963-1820

\section{Éditeur}

Les éditions de la Maison des sciences de l'Homme

\section{Référence électronique}

Catherine Colliot-Thélène, «L'interprétation des droits de l'homme : enjeux politiques et théoriques au prisme du débat français », Trivium [En ligne], 3 | 2009, mis en ligne le 15 avril 2009, consulté le 23 septembre 2020. URL : http://journals.openedition.org/trivium/3290; DOI : https://doi.org/10.4000/ trivium. 3290

Ce document a été généré automatiquement le 23 septembre 2020.

\section{cc)}

Les contenus des la revue Trivium sont mis à disposition selon les termes de la Licence Creative Commons Attribution - Pas d'Utilisation Commerciale - Pas de Modification 4.0 International. 


\title{
L'interprétation des droits de l'homme : enjeux politiques et théoriques au prisme du débat français
}

\author{
Catherine Colliot-Thélène
}

1 La littérature de langue française sur les droits de l'homme s'est accumulée depuis les années 1980, après une longue période pendant laquelle la notion avait été soit négligée, soit tenue en suspicion par les discours théoriques dominants. Au plan philosophique, durant environ deux décennies, la critique du sujet et de la métaphysique de la subjectivité, dans laquelle se retrouvaient les disciples de Heidegger, un certain marxisme et diverses variétés de cette mouvance que l'on désigne sous le nom de structuralisme, avaient conjugué leurs effets pour rendre douteuse la consistance théorique de l'idée des droits de l'homme. S'y ajoutaient, du côté de la pensée de gauche, la méfiance à l'égard du droit en général, dont beaucoup considéraient avec Marx que sa fonction essentielle était de servir un État lui-même au service de la classe dominante, ainsi que la polarisation politique extrême des années 1960 et 1970, au regard de laquelle les droits de l'homme, du fait de leur universalité et par conséquent de leur neutralité par rapport aux clivages politiques et sociaux, étaient supposés ne pas avoir de pertinence politique. Du côté des juristes enfin, un autre élément contribuait encore au discrédit des droits de l'homme, à savoir la sévère critique que l'historien du droit Michel Villey faisait de la notion de droit subjectif dans ses cours et ses écrits. S'appuyant sur une impressionnante érudition et jouant avec virtuosité de ses talents de polémiste, Villey a consacré un ouvrage spécifique à la critique de « l'idéologie droits de l'homme ${ }^{1}$ » dont l'influence s'est étendue bien au-delà $\mathrm{du}$ cercle des juristes et des historiens du droit. Dans un livre récent ${ }^{2}$, le philosophe Vincent Descombes cite encore la formule lapidaire par laquelle Villey résumait sa thèse fondamentale: "le droit ne connaît pas de sujets, il ne connaît que des attributaires ", qu'il reprend à son compte dans le cadre d'une critique, d'inspiration wittgensteinienne, des différents avatars de la notion de sujet. Nonobstant la différence 
du cadre théorique, Descombes prétend comme Villey résoudre la « querelle des droits subjectifs » en renonçant à la notion de sujet de droits.

2 Les préventions, théoriques et politiques, étant si fortes, d'où vient que la thématique des droits de l'homme, depuis le tournant des années 1970 et 1980 du siècle dernier et jusqu'à aujourd'hui, ait donné lieu à une floraison d'articles et d'ouvrages? La critique, certes, ne s'est pas entièrement tue. Dès 1980, Marcel Gauchet la réactualisait en soutenant, contre ceux qui cherchaient dans les droits de l'homme un substitut à un discours politique de gauche épuisé, que «les droits de l'homme ne sont pas une politique $^{3}$ ». Quelques années plus tard, Bertrand Binoche rappelait les différentes variantes des critiques des droits de l'homme depuis l'époque des Révolutions française et américaine ${ }^{4}$. Et Vincent Descombes, on l'a dit, poursuit cette querelle au début des années 2000. On peut même dire que cette critique s'est formulée durant les trente dernières années de manière plus explicite que dans les décennies antérieures, où l'idée des droits de l'homme paraissait généralement si désuète que l'on éprouvait rarement la nécessité de s'y attarder. Mais si cette critique est devenue plus explicite, c'est parce qu'elle réagissait à une conjoncture politico-théorique nouvelle, dans laquelle les droits de l'homme avaient de nouveau acquis le statut d'objet politique. De là vient que les critiques récentes de la notion, si elles ne se privent pas à l'occasion de puiser dans le stock d'arguments disponibles dans des critiques anciennes, sont cependant nouvelles et leurs arguments, en partie du moins, originaux, comme l'est le contexte où les droits de l'homme ont resurgi comme enjeu de débats et de luttes politiques.

3 Si l'on peut considérer qu'il s'est produit à partir de la fin des années 1970 et, de manière plus prononcée, du début des années 1980, une inflexion significative dans l'histoire idéologique et théorique des droits de l'homme en France, inflexion qui s'est traduite par une revalorisation de leur signification politique après des décennies d'indifférence ou de mépris, les éléments du contexte politique et social qui nourrissent depuis ce tournant l'intérêt continu pour les droits de l'homme se sont aussi modifiés au long des années qui ont suivi. Des préoccupations nouvelles, nées de l'actualité politique, sont venues se substituer ou s'ajouter à celles qui avaient suscité le renouveau des années 1980. On se contentera ici d'une énumération rapide des éléments qui, soit conjointement, soit successivement, ont déterminé ce contexte et son évolution.

4 Ce fut d'abord, à la fin des années 1970, le soutien aux luttes des dissidents des pays soviétiques et la critique des régimes totalitaires ${ }^{5}$. Dix ans plus tard, le bicentenaire de la Révolution française, dont le hasard historique a voulu qu'il coïncidât avec l'effondrement du régime soviétique, fournit l'occasion de revisiter les sources. À quoi correspondit la publication des textes de référence ainsi que de commentaires, directs ${ }^{6}$ ou indirects ${ }^{7}$. Dans la foulée de 1989 se sont produites, à l'échelle du monde, des crises politiques pour lesquelles les grilles de lecture classiques (de la gauche comme de la droite) ne permettaient plus, ou difficilement, de prendre parti, et dans lesquelles se commettaient des massacres (l'éclatement de la Yougoslavie et ce qui s'en est suivi). Ce fut la montée de "l'humanitaire", qui ne pouvait qu'invoquer les droits de l'homme tout en les dépolitisant dans une logique compassionnelle qui servait avant tout à occulter l'impuissance du politique ${ }^{8}$. Parallèlement, la question de l'immigration, dont les politiques tacticiennes des partis avaient fait un enjeu central des affrontements électoraux de l'hexagone, a ajouté une dimension nouvelle à la discussion sur les droits de l'homme. Les politiques de plus en plus restrictives de la France, mais aussi de 
l'ensemble des pays européens ainsi que des États-Unis dans le domaine de l'immigration se sont accompagnées de mesures policières envers les immigrés qui justifiaient de reprendre la question naguère soulevée par Hannah Arendt à propos de la situation des apatrides: l'effectivité des droits de l'homme n'est-elle pas conditionnée par le statut de citoyen? En dernier lieu, le développement de dispositifs judiciaires supra-nationaux, permanents (CIPJ, CPI) ou temporaires (TPIY, TPIR), qui statuent en référence à des textes fondés sur les principes des droits de l'homme, a produit, du moins chez les juristes, une réflexion sur les promesses et les difficultés de la mondialisation du droit ${ }^{9}$.

5 Chacun de ces éléments a contribué, dans les trente dernières années, à nourrir la réflexion sur les droits de l'homme, induisant des modifications plus ou moins appuyées du cadre interprétatif en fonction duquel leur signification était analysée. L'histoire récente de la problématisation des droits de l'homme a croisé ainsi, successivement ou parallèlement, la reconsidération des rapports entre politique et morale, le débat sur l'individualisme, la question de la citoyenneté ou encore la question du rapport entre Universel et particulier (les droits de l'homme sont-ils une idéologie occidentale?). Faute de pouvoir retracer les différentes étapes de cette histoire dans cette introduction, on rappellera seulement deux aspects du débat français qui expliquent les spécificités nationales du traitement de cette question d'intérêt universel.

\section{L'interprétation libérale}

6 Le premier est la surdétermination de l'interprétation de la signification des droits de l'homme par l'opposition qui a structuré les clivages politico-théoriques en France tout au long $\mathrm{du} \mathrm{xx}^{\mathrm{e}}$ siècle, entre les penseurs libéraux et une gauche qui se recommandait peu ou prou du marxisme. Vers la fin des années 1970 et plus encore dans la décennie qui a suivi, les libéraux se sont saisi de l'opportunité d'un moment qui voyait se conjuguer la perte de crédibilité de la doxa révolutionnaire, l'essoufflement de la configuration théorique qui avait porté la récusation de la figure du sujet et, enfin, la célébration de la Révolution française, pour proposer une grille d'interprétation radicalement nouvelle de l'histoire politique de la France des deux derniers siècles. Les travaux de François Furet, de Marcel Gauchet, et le Dictionnaire critique de la Révolution française, publié en 1988, ont fixé les thèmes de cette relecture libérale de la Révolution française et de ses effets. Il allait de soi que l'interprétation de la Déclaration des droits de l'homme devait constituer une pièce centrale de cette lecture.

7 Le noyau de cette lecture nouvelle résidait dans la dénonciation du mythe de la Nation souveraine, considérée par l'ensemble de ces auteurs comme un schème symbolique hérité du centralisme monarchique. Ce mythe est selon eux à l'origine du légicentrisme de la tradition politique française, pour lequel Rousseau aurait fourni l'assise théorique. En posant que la loi est l'expression de la volonté générale (DDH, art. 6), la Déclaration ouvrait la voie à la revendication d'une participation du peuple à l'exercice du pouvoir. Que les constituants se soient ralliés, malgré Rousseau, à la nécessité de la représentation, n'a pas empêché cette exigence de participation d'alimenter, durant les deux siècles qui ont suivi, une contestation récurrente des pouvoirs établis, à laquelle ces auteurs imputent l'instabilité permanente de la vie politique française. Cette logique participative s'accompagne d'une sous-estimation de la fonction protectrice de 
la Déclaration des droits. Dès lors en effet que la loi est conçue comme l'expression de la volonté générale, il n'est nulle raison justifiable de doter les individus de droits dont ils puissent se prévaloir contre elle. Le légicentrisme rousseauiste est ainsi rendu responsable $\mathrm{du}$ « radicalisme démocratique mal entendu ${ }^{10}$ » de la tradition politique française, en même temps que de la complaisance de la Gauche envers le totalitarisme des régimes soviétiques.

Cette lecture libérale de la Déclaration de 89 est sous-tendue par une comparaison entre celle-ci et ses analogues américains, sur le fond d'une opposition générale entre la tradition démocratique française et la tradition américaine, qui, sur tous les points, tourne à l'avantage de la seconde. En gros, les échecs et les impasses de la vie politique française sont imputés à l'héritage monarchique, que le jacobinisme se serait contenté de revêtir de vêtements nouveaux en substituant la souveraineté de la Nation à celle du Roi, et qui aurait continué à formater la conception française de la démocratie jusque dans les dernières décennies $\mathrm{du} \mathrm{xx}^{\mathrm{e}}$ siècle. Au regard de la Révolution américaine, instituée en norme de la modernité justement comprise, la Révolution française et la tradition démocratique qu'elle a initiée apparaissent définitivement passéistes. Quand la gauche française a redécouvert les droits de l'homme après les avoir longtemps négligés, les libéraux lui ont reproché d'accorder à la déclaration de ces droits une fonction fondatrice pour les institutions et d'appuyer sur eux l'exigence participative du citoyen. Ce que cette gauche s'obstinait selon eux à ignorer est la distinction requise entre la sphère du privé et du public, et par conséquent la " différence du politique ${ }^{11}$ ", c'est-à-dire l'extériorité du pouvoir par rapport à la société. À quoi les libéraux opposaient la signification politique des droits de l'homme en leur interprétation américaine, qui tient au contraire en cela qu'ils ne sont pas destinés à fonder le pouvoir, mais à en délimiter les bornes, donc à protéger les individus contre des instances de gouvernement dont l'existence est certes nécessaire, mais qui demeurent et doivent demeurer distinctes de la société. Les révolutionnaires américains n'ont pas méconnu les virtualités oppressives du pouvoir. À la différence des français toutefois, ce n'est pas en brouillant la différence entre le pouvoir et la société qu'ils ont cherché à s'en prémunir, mais en aménageant les institutions de telle sorte que des organes de pouvoir différenciés se limitent et se contraignent mutuellement. Montesquieu les inspirait, plutôt que Rousseau, et une judicieuse architecture institutionnelle, dont la conception était conduite par des considérations pragmatiques, leur est apparue une plus sûre garantie des droits de l'homme que l'élévation du peuple au statut de législateur ${ }^{12}$.

\section{Les relectures de gauche}

9 L'interprétation libérale a fait date et elle a aujourd'hui encore ses défenseurs. Mais la dissidence des pays de l'est, l'effondrement du système soviétique et le bicentenaire de la Déclaration des droits de l'homme ont suscité également des réflexions nouvelles de la part de théoriciens de gauche. Bien que moins médiatisées que celles des libéraux, ces réflexions ouvraient elles aussi des perspectives inédites. Le texte de Claude Lefort et celui d'Étienne Balibar, tous deux présentés en traduction allemande dans ce dossier, en sont de bons exemples. Ces textes parlent d'eux-mêmes, et il est inutile d'en résumer toutes les thèses. On se contentera de souligner les raisons pour lesquelles, vingt ans et 
trente ans après leur publication, ils nous paraissent se recommander aujourd'hui à notre attention.

À l'instar des libéraux, Lefort a élaboré son interprétation des droits de l'homme à partir de la critique du totalitarisme, et comme eux, il réagissait à l'usage compensatoire que la gauche et l'extrême gauche faisait de la référence à ces droits dans la seconde partie des années 1970. Mais malgré ses distances par rapport à Marx, et plus encore par rapport au marxisme du Parti communiste ou de l'extrême gauche, il inscrivait ses analyses dans l'horizon d'une logique démocratique comprise comme processus d'émancipation. Il est remarquable que l'on ne trouve pas chez lui l'opposition entre la tradition française et la tradition américaine. C'est que son propos n'est pas de fixer une orthodoxie concernant le juste aménagement des institutions, mais de souligner le caractère radicalement novateur du principe même de la "déclaration». L'énonciation des droits importe selon lui plus encore que leur contenu. Par le fait de cette énonciation, les droits dont jouissent les hommes en société se trouvent en effet référés non plus, comme jadis, à un ordre social immuable ou à un contrat entre le pouvoir et les assujettis, mais à l'acte de leur revendication. Si Lefort, comme les libéraux, relativise la rupture constituée par la Révolution française en reconnaissant une certaine continuité entre celle-ci et la centralisation monarchique qui l'a précédée, cette continuité ne se situe pas selon lui au plan symbolique, dans le mythe de la nation souveraine, mais dans les structures administratives d'un pouvoir territorial qui s'exerçait par le moyen de la loi. La Révolution n'a pas institué l'État de droit, mais elle a, à l'intérieur d'un État de droit déjà existant, modifié en profondeur les rapports entre le pouvoir et le droit. La Déclaration des droits de l'homme a été selon Lefort l'instrument décisif de cette mutation. Contre la lecture de Marx, Lefort soutient que l'homme des droits de l'homme n'est pas le propriétaire, c'est-à-dire l'individu égoïste de la société bourgeoise. Mais il n'y voit pas non plus l'individu qui, méconnaissant la différence entre l'ordre du privé et celui du public, confond la liberté avec la participation à la puissance du collectif. Référé à l'entité indéterminée qu'est «l'homme » en général, le droit constitue par rapport au pouvoir une extériorité irréductible, un «foyer de légitimité immaîtrisable ${ }^{13}$ » qui implique que l'ordre établi puisse être toujours mis en question. Il ouvre ainsi sur une histoire dans laquelle «les droits acquis sont nécessairement appelés à soutenir des droits nouveaux ${ }^{14} »$.

11 Balibar conteste aussi la lecture marxienne de la Déclaration de 89, dans une perspective un peu différente de celle de Lefort, qui aboutit cependant à des conclusions convergentes sur un point essentiel. L'erreur de Marx a été selon lui de reprendre à son compte la compréhension libérale du couple homme-citoyen en l'interprétant comme une opposition. Or c'est précisément l'identification des deux concepts qui constitue la grande innovation du texte, ce qui en fait le caractère révolutionnaire. Loin d'être les droits garantis à l'homme à titre d'individu privé, les droits de l'homme sont des droits pleinement politiques. Plus précisément: ils manifestent l'idée d'un droit des hommes, de tous les hommes, omnes et singulatim, à la politique. Cette équation en présuppose une autre, que Balibar résume dans un mot valise : l'égaliberté. À l'encontre de ce qui est devenu un axiome du libéralisme des XIX et $\mathrm{xx}^{\mathrm{e}}$ siècle (axiome que le socialisme a avalisé, lors même qu'il en tirait de tout autres conséquences), la logique de la Déclaration n'opposait pas plus liberté et égalité qu'elle ne distinguait l'homme du citoyen. Du fait qu'elle posait au contraire que liberté et 
égalité sont coextensives, elle enclenchait un processus de politisation des revendications de droits dans la dynamique de laquelle se sont inscrites toutes les luttes d'émancipation des deux siècles suivants. Quand Balibar écrit, faisant écho à Marx, que "l'émancipation des opprimés ne peut être que leur œuvre propre ", il rejoint l'idée avancée par Lefort d'un «foyer de légitimité immaitrisable » des régimes démocratiques modernes. Les deux auteurs soulignent que ce principe a pour conséquence une instabilité structurelle de ces régimes, instabilité qu'il est non seulement illusoire, mais dangereux de vouloir supprimer, dans la mesure où elle résulte de ce qui est le moment proprement démocratique de ces régimes. Balibar explore en outre les contradictions inhérentes au concept post-révolutionnaire du politique, écartelé entre le réquisit de l'institution et la légitimation de la contestation de toute forme figée de celle-ci (ou, dans les termes qui sont les siens : entre logique de la constitution et logique de l'insurrection), et il développe les apories d'une citoyenneté tendanciellement universelle et qui semble pourtant ne pouvoir prendre corps que dans des formes institutionnelles qui déplacent l'exclusion sans jamais la supprimer.

Quelques mots sur mon propre texte, pour dire simplement les liens qu'il entretient avec les deux précédents. Il s'en distingue par son point de départ, qui n'est pas l'interprétation de la Déclaration des droits de l'homme, mais une tentative de réhabilitation de la notion de droit subjectif contre l'interdit prononcé à son encontre par Michel Villey et, bien plus tôt, par certains théoriciens du droit ${ }^{15}$. Il les rejoint pourtant - et l'on voit ici combien la signification des droits de l'homme est liée à l'originalité de la figure juridique du sujet de droit - en soulignant l'historicité du sujet politique moderne ainsi que celle, corrélative, de la démocratie. La réalité de celle-ci ne se situe pas dans les structures d'un régime de gouvernement (bien que ces structures ne lui soient pas indifférentes), mais dans la dynamique de leur contestation toujours renouvelée et des transformations institutionnelles qui résultent de cette contestation. En d'autres termes: ce sont les luttes que les exclus du pouvoir, quels qu'ils soient (ouvriers, femmes, noirs américains, immigrés, etc.), mènent, au nom de l'égalité, contre les pouvoirs institués, qui sont le moteur de la démocratie. Cette proposition ne fait que reprendre un thème déjà décliné par Lefort, Balibar, ainsi d'ailleurs que par Rancière ${ }^{16}$. Sans doute peut-elle être considérée comme une expression de « radicalisme démocratique ». Mais le déplacement qui s'est opéré il y a une vingtaine d'années de la question de la révolution à celle de la citoyenneté a eu pour conséquence que ce radicalisme ne vise plus l'éradication du pouvoir, non plus que sa conquête ou la participation à celui-ci, mais qu'il prend acte de son irréductible extériorité. Le pouvoir est désormais conçu comme le partenaire nécessaire d'une relation conflictuelle qui ne peut connaitre que des solutions provisoires, le destinataire de revendications qui le présupposent en même temps qu'elles ne cessent de le mettre en question.

\section{Droits de l'homme, droits subjectifs : leur avenir}

$13 \mathrm{Au}$ vu de ce qui précède, on ne s'étonnera pas que la réflexion sur les droits de l'homme, que les commentaires des Déclarations révolutionnaires avaient tout naturellement liée à la compréhension de la citoyenneté, ait croisé ces dernières décennies l'analyse des figures contemporaines de l'exclusion : celle des sans logis, des sans travail, et aussi celle de l'immigré «sans papier ». C'est là le second aspect du 
débat français contemporain sur les droits de l'homme, qui s'indique en filigrane dans les textes ici traduits. Parmi tous les «sans part " (selon la formule de Rancière) dont les mobilisations revendicatrices contribuent à redéfinir la compréhension de la citoyenneté, l'immigré est sans doute aujourd'hui la figure la plus représentative ${ }^{17}$. La création d'une citoyenneté européenne par le Traité de Maastricht en 1992 a ébranlé le lien d'implication réciproque entre citoyenneté et nationalité qui avait déterminé la compréhension citoyenne durant près de deux siècles. Mais, parce que cette citoyenneté européenne est souvent pensée comme une reproduction, à échelle élargie, du modèle de la citoyenneté nationale, elle n'intègre elle aussi qu'en excluant. C'est le sort des apatrides durant l'entre-deux-guerres et durant la Seconde Guerre mondiale qui avait amené Hannah Arendt à formuler le concept d'un "droit à avoir des droits ", c'est-à-dire d'un droit à l'inclusion politique qui apparaît comme la condition d'effectivité des droits de l'homme. C'est la condition du demandeur d'asile ou du travailleur immigré sans papier qui donne de nos jours à ce concept une actualité renouvelée et incite à y voir l'expression la plus radicale, mais aussi la plus fidèle de la citoyenneté de l'époque révolutionnaire et post-révolutionnaire.

Les lectures libérales de la Déclaration des droits de l'homme voulaient fermer une page d'histoire, celle des Révolutions. Les relectures de gauche, en soulignant la signification émancipatrice de la revendication de droits qui ne se réclament pas du passé, mais d'une nature à laquelle seuls les droits conquis, généralement de haute lutte, peuvent donner une détermination qui lui fait originairement défaut, ouvrent au contraire vers l'avenir. La révolution démocratique n'est jamais achevée, parce que le sens de la démocratie ne peut pas s'épuiser dans des agencements institutionnels. Certains sont certainement préférables à d'autres, du point de vue de leur stabilité peut-être (mais il est des régimes dictatoriaux qui ont la vie longue, ce qui ne suffit pas à les justifier), du point de vue des possibilités qu'ils autorisent pour une opposition sûrement, mais aucun ne garantit définitivement contre les dérives du pouvoir. La vitalité de la démocratie, sa capacité à intégrer des transformations sociales et culturelles que l'on ne peut anticiper, dépend cependant au moins autant de l'action revendicative des exclus que de la "gouvernance ", bonne ou mauvaise, des tenants du pouvoir ${ }^{18}$. L'un des avantages d'une conception de la démocratie indexée sur les pratiques citoyennes plutôt que sur les formes des régimes politiques, est qu'elle ouvre la citoyenneté sur des espaces autres que nationaux. Sans doute du fait de la prégnance de la tradition républicaine française, pour laquelle l'État-nation reste le cadre obligé de la "communauté des citoyens ${ }^{19}$ ", les philosophes français dans leur ensemble se sont jusqu'à présent peu intéressés à la construction européenne, et encore moins aux dimensions politiques, juridiques et judiciaires de la mondialisation. La jonction entre leur réflexion et celles que certains juristes et politistes développent déjà à ce propos reste à faire. J'ai tendance à penser - mais je n'engage ici que moi - que c'est précisément cette jonction qui permettra de déployer toutes les ressources que recèle la figure du citoyen sujet de droits, faisant ainsi accomplir quelque pas de plus à la dissociation déjà amorcée entre citoyenneté et nationalité. 


\section{BIBLIOGRAPHIE}

\section{Sélection thématique de textes français :}

\section{Sources :}

Delmas-Marty, M. / Lussac de Leyssac, C. (éd.) (1996) : Libertés et droits fondamentaux, Paris.

Jaume, L. (éd.) (1989) : Les Déclarations des droits de l'homme, Paris.

Rials, S. (éd.) (1988) : La Déclaration des droits de l'homme et du citoyen, Paris.

\section{Littérature française :}

Badie, B. (2002) : La diplomatie des droits de l'homme, Paris, Fayard.

Balibar, É. (2002-2003) : Nouvelles réflexions sur l'égaliberté (Deux leçons, inédit).

Balibar, É. (2002) : Droit de cité, Paris, Presses Universitaires de France.

Balibar, É. (1989) : « La proposition de l'égaliberté », in : Les conférences du Perroquet, $\mathrm{n}^{\circ} 22$, novembre 1989.

Binoche, B. (1989) : Critiques des droits de l'homme, Paris, Presses Universitaires de France.

Binoche, B. / Cléro, J-P. (2007) : Bentham contre les droits de l'homme, Paris, Presses Universitaires de France.

Bourgeois, B. (1990) : Philosophie et droits de l'homme (de Kant à Marx), Paris, Presses Universitaires de France.

Delmas-Marty, M. (2007) : La refondation des pouvoirs, Paris, Seuil.

Delmas-Marty, M. (2006) : Le pluralisme ordonné, Paris, Seuil.

Delmas-Marty, M. (2004) : Le relatif et l'universel, Paris, Seuil.

Delmas-Marty, M. (éd.) (1989) : Raisonner la raison d'État. Vers une Europe des droits de l'homme, Paris, Presses Universitaires de France.

Descombes, V. (2004) : Le complément de sujet, Paris, Gallimard.

Gauchet, M. (1989) : La Révolution des droits de l'homme, Paris, Gallimard.

Gauchet, M. (1988) : « Droits de l'homme », in : Furet, F. / Ozouf, M. (éd.), Dictionnaire critique de la Révolution française, Paris, Flammarion, p. 685-695.

Gauchet, M. (1980) : «Les droits de l'homme ne sont pas une politique », in : Le Débat, $\mathrm{n}^{\circ}$ 3, juilletaoût 1980, p. 3-21.

Kervégan, J.-F. (1995) : « Les droits de l'homme », in : Kambouchner, D. (éd.), Notions de Philosophie, t. II, Paris, Gallimard, p. 637-696.

Lefort, C. (1981) : «Droits de l'homme et politique », in : Libre, n 7, Paris (republié dans Lefort, C., L'invention démocratique, Paris, Le livre de poche, 1981 [1994], p. 45-86). 
Lefort, C. (1980) : « La pensée politique devant les droits de l'homme », Europa, revue d'Études interdisciplinaires, Montréal (republié dans Lefort, C., Le temps présent, Paris, Belin, 2007, p. 405-421).

Lochak, D. (2007) : Face aux migrants : État de droit ou état de siège?, entretien avec Bertrand Richard, Paris, Textuel.

Lochak, D. (2002) : Les droits de l'homme, Paris, La Découverte.

Rancière, J. (2004) : Aux bords du politique, Paris, Gallimard.

Sassen, S. (2006) : Territory, Authority, Rights, Princeton, Princeton University Press.

Schnapper, D. (1994) : La communauté des citoyens, Paris, Gallimard.

Villey, M. (2003) : La formation de la pensée juridique moderne, Paris, Presses Universitaires de France.

Villey, M. (1983) : Le droit et les droits de l'homme, Paris, Presses Universitaires de France.

\section{NOTES}

1. Villey (1983).

2. Cf. Descombes (2004).

3. Gauchet (1980).

4. Cf. Binoche (1989).

5. Cf. Lefort (1980), Lefort (1981).

6. Cf. Gauchet (1988), Balibar (1989).

7. Cf. Bourgeois (1990).

8. Cf. Badie (2002).

9. Cf. Delmas-Marty (2004), Delmas-Marty (2006), Delmas-Marty (2007).

10. Gauchet (1989), p. 85.

11. Ibid., p. 79.

12. Pour une interprétation plus nuancée de la différence entre les Déclarations des droits françaises et américaines, cf. Kervégan (1995).

13. Lefort (2007), p. 64.

14. Ibid., p. 67.

15. Il existe bien en France des travaux de juristes et d'historiens du droit sur la notion de droits subjectifs. Du côté de la philosophie politique en revanche, la réflexion s'est focalisée sur la question des droits de l'homme et de leur rapport avec les droits du citoyen. Il y a peu de choses sur l'originalité historique de la figure théorique du droit subjectif et ce qu'elle implique quant aux modalités de l'inclusion sociale.

16. Cf. Rancière (2004)

17. Cf. Balibar (2002) notamment, parmi les textes rassemblés dans ce recueil: «Ce que nous devons aux "Sans-Papiers" » et « Une citoyenneté européenne est-elle possible? ».

18. Cf. en ce sens Sassen (2006), p. 290-292. Dans le cadre d'une réflexion sur la complexité de la citoyenneté aujourd'hui, ainsi que sur la formation des sujets porteurs de droits, Saskia Sassen développe la thèse selon laquelle «la citoyenneté est en partie produite par les pratiques des exclus", lesquelles ouvrent la voie à «des droits pour des acteurs et des problèmes non formalisés, en particulier quand la maîtrise de l'État-nation sur les questions d'identité et d'appartenance est affaiblie par des tendances sociales, économiques, politiques et subjectives » (op. cit., p. 290).

19. Schnapper (1994). 
INDEX

Mots-clés : droits de l'homme, droits (subjectifs)

Schlüsselwörter : Menschenrechte, Rechte (subjektive)

\section{AUTEUR}

\section{CATHERINE COLLIOT-THÉLÈNE}

Catherine Colliot-Thélène est professeur de philosophie à l'Université de Rennes I. Pour plus d'informations, voir la notice suivante. 